\title{
Primer Registro de Diaphania hyalinata (Linnaeus) y $D$. nitidalis (StolI) (Lepidoptera: Crambidae) sobre Melothria pendula L. (Cucurbitaceae) en el estado Lara, Venezuela
}

\author{
First record of Diaphania hyalinata (Linnaeus) and D. nitidalis \\ (Stoll) (Lepidoptera: Crambidae) on Melothria pendula L. \\ (Cucurbitaceae) in the Lara state, Venezuela
}

\section{Tarcisio José Capote Luna ${ }^{1}$, Evelin Antonieta Arcaya Sánchez ${ }^{1 *}$ y Dilcia María Hernández Juárez ${ }^{1}$}

\footnotetext{
${ }^{1}$ Universidad Centroccidental "Lisandro Alvarado" (UCLA), Decanato de Agronomía, Departamento de Ciencias Biológicas. Lara, Venezuela.
}

\author{
*Autor para correspondencia: \\ evearcaya@gmail.com \\ Conflicto de interés: \\ Los autores declaran no tener \\ conflicto de interés. \\ Licencia: \\ Artículo publicado en acceso \\ abierto con una licencia Creative \\ Commons CC-BY \\ Historial: \\ Recibido: $11 / 09 / 18$; \\ Aceptado: 05/07/19 \\ Periodo de Publicación: \\ Julio-Diciembre de 2019
}

\begin{abstract}
RESUMEN
Diaphania hyalinata (Linnaeus) y D. nitidalis (Stoll) (Lepidoptera: Crambidae) son especies fitófagas de importancia económica en cucurbitáceas. En este estudio se reportan por primera vez para el estado Lara, Venezuela a $D$. hyalinata y $D$. nitidalis sobre Melothria pendula L. (Cucurbitaceae) como planta hospedante natural y se amplía el registro del área de distribución de $D$. nitidalis para éste estado.

Palabras clave: Barrenador del pepino, plagas en cucurbitáceas, distribución de Diaphania, gusano del melón
\end{abstract}

\begin{abstract}
Diaphania hyalinata (Linnaeus) and D. nitidalis (Stoll) (Lepidoptera: Crambidae) are phytophagous species of economic importance in cucurbits. This study reports for the first time for Lara state, Venezuela on D. hyalinata and D. nitidalis on Melothria pendula L. (Cucurbitaceae) as a natural host plant and the distribution of $D$. nitidalis for this state is extended.
\end{abstract}

Key words: Pickleworm, pests in cucurbits, distribution of Diaphania, melonworm

\section{INTRODUCCIÓN}

El gusano del melón, Diaphania hyalinata (Linnaeus) (Lepidoptera: Crambidae) se reporta durante los meses de verano para algunos estados de Norteamérica (Brou, 2016), en forma permanente en Centroamérica, Sudamérica, el Caribe (Saunders, Coto y King 1998) y Paraguay (PYBIO, 2018). En Venezuela, D. hyalinata está registrada para los estados Amazonas, Aragua, Bolívar, Carabobo, Distrito Capital, Falcón, Guárico, Lara, Mérida, Miranda, Portuguesa, Táchira, Trujillo, Yaracuy (Clavijo-Albertos y Arias-Celis, 2017). Es una especie fitófaga que consume el follaje sin atacar la nervadura de la hoja y raspa la superficie del fruto, prefiere como hospedante el calabacín (Curcubita pepo L.) y se le considera el principal insecto plaga de las cucurbitáceas en el estado de Florida, USA (Capinera, 2017b). En Venezuela, se reporta como insecto plaga en el cultivo de melón para los estados Lara y Zulia (Geraud-Pouey, Chirinos, Peña y Arcilla, 1998; Salas, 2011).

El barrenador del pepino, D. nitidalis (Stoll) (Lepidoptera: Crambidae) en su estado adulto es una polilla que se encuentra distribuida a lo largo del continente desde el sureste de los Estados Unidos de América, Costa Rica, Perú, Brasil hasta Argentina (Brito, Costa, Maziero, Brito é Dörr, 2004), (Cordo, Logarzo, Braun y Di Lorio, 2004; Janzen \& Hallwachs, 2009; Arévalo, 2016, VanDick, 2018).

Se ha evaluado la preferencia de alimentación de larvas del barrenador, empleando hojas de cucurbitáceas silvestres como Melothria pendula L. y Momordica charantia L. (Cucurbitaceae). Las larvas alimentadas con $M$. pendula tuvieron un 
adecuado desarrollo, mientras que con M. charantia no sobrevivieron (Elsey, Pena \& Waddill, 1985). D. nitidalis se reporta en cultivares de calabazas y calabacines como hospedantes apropiados y en $M$. pendula como el principal hospedante silvestre de la especie en el estado de Florida, USA (Capinera, 2017a). En Venezuela, D. nitidalis se encuentra entre los insectos plagas reportados en el cultivo de melón (Cucumis melo L.) y pepino (Cucumis sativus L.) (Salas, 2011; Acevedo et al., 2012; Ruíz y Russián, 2017).

El pepino de culebra (M. pendula) es una de las 12 especies del género Melothria L. (The Plant List, 2013). Es una enredadera rastrera o trepadora que se encuentra en todo el continente desde el sureste de los Estado Unidos de América, México, Colombia, Brasil hasta Argentina (Flora do Brasil, 2017; Instituto de Ciencias Naturales, 2017; Flora Argentina, 2018; Centro de Investigación Científica de Yucatán, 2018; USDA, 2018).

En Venezuela, se ha reportado su presencia entre 100 a 1600 msnm, en los estados Amazonas, Apure, Aragua, Barinas, Carabobo, Delta Amacuro, Distrito Capital, Falcón, Guárico, Lara, Mérida, Miranda Monagas, Nueva Esparta, Sucre, Táchira, Trujillo, Yaracuy, Zulia (Hokche, Berry y Huber, 2008) y Cojedes (Delascio-Chitty y López, 2007). Produce un fruto que semeja una patilla o pepino en miniatura, sub-globoso hasta elipsoidal, apiculado, de 1 a $3 \mathrm{~cm}$ de largo y 0,8 a $2 \mathrm{~cm}$ de ancho, de color verde en la fase inmadura, negro o purpúreo obscuro estando maduro (Schnee, 1984; Lira, 2001). Estudios bromatológicos indican que es una especie silvestre no domesticada con potencial para ser empleada en la alimentación humana por el agradable sabor de sus frutos y contenido de $56,8 \%$ de carbohidratos y $12,6 \%$ de proteínas (ArzateFernández y Grenón-Cascales, 2002).

En la presente investigación se registra por primera vez la presencia de $D$. hyalinata y $D$. nitidalis sobre M. pendula L. en el estado Lara, Venezuela.

Entre los meses de enero y marzo de 2018 se observó la presencia de larvas de lepidópteros alimentándose de frutos y hojas del pepino de culebra en un cultivo de vid Criolla Negra, un cultivar nativo de Vitis vinifera $x$ Vitis caribaea (Olmo, 1968), del Instituto de la Uva del Decanato de Agronomía de la Universidad Centroccidental Lisandro Alvarado (UCLA) (100 01'09' 'N,
69017'18' 'O), 500 m, Tarabana, municipio Palavecino, estado Lara, Venezuela.

Las muestras botánicas se tomaron empleando las técnicas tradicionales de herborización y se depositaron en el Herbario José Antonio Casadiego (UCOB), Decanato de Agronomía de la UCLA. Para las muestras entomológicas se recolectaron frutos y hojas del pepino de culebra. Este material se introdujo en envases plásticos de $0,5 \mathrm{~L}$ de capacidad, rotulados con una etiqueta que indicó el lugar, fecha, la planta hospedante y el nombre del recolector.

Los envases utilizados para la recolección fueron previamente preparados con una abertura en su tapa, cubierta por tela tipo tul, con el propósito de una adecuada ventilación. Una vez finalizada la recolecta, los envases plásticos fueron trasladados al laboratorio de investigación de Entomología del Decanato de Agronomía de la UCLA, ubicado en Tarabana, Estado Lara.

En el laboratorio, los frutos y hojas se colocaron en envases de vidrio de capacidad 3,875 L, tapados con tela tipo tul. Se colocaron en una sala de cría a $29 \pm$ $1^{\circ} \mathrm{C}, 70 \pm 5 \%$ HR y 12:12 (D:N) horas de fotoperiodo. Los adultos obtenidos se montaron, etiquetaron y depositaron en el Museo de Entomología José Manuel Osorio (MJMO), Decanato de Agronomía de la UCLA.

Los lepidópteros se identificaron por comparación morfológica siguiendo los trabajos de Janzen y Hallwachs (2009), la clave del género Diaphania Hübner (Clavijo, Munroe y Arias, 1995; Arias y Clavijo, 2001).

El pepino de culebra fue identificado por comparación con fotografía, descripción y clave de la familia Cucurbitaceae (Hardy, 1976). Las fotografías fueron realizadas con una cámara fotográfica digital marca Canon PowerShot SD750®.

Del muestreo se obtuvieron un total de 10 ejemplares. Los lepidópteros encontrados fueron identificados como $D$. hyalinata y $D$. nitidalis (Figura $1 \mathrm{~A}-\mathrm{B})$, mientras que la planta fue identificada como M. pendula (Figura 2). Este trabajo reporta por primera vez a $D$. hyalinata y $D$. nitidalis como especies asociadas a M. pendula en el estado Lara y se realiza un nuevo registro de $D$. nitidalis para el mismo estado lo cual constituye una fuente de 
información valiosa para ampliar el conocimiento de esta especie sobre la flora hospedante, de manera que con este nuevo aporte se puede asegurar con certeza la presencia de tres especies del género Diaphania considerando el registro previo de $D$. indica en un cultivo hidropónico de pepino en la misma localidad (Arcaya, Díaz y Paz, 2004). Además, se enriquecen los datos de distribución geográfica para Sudamérica al registrar por primera vez a la especie en la región centro occidental de Venezuela.
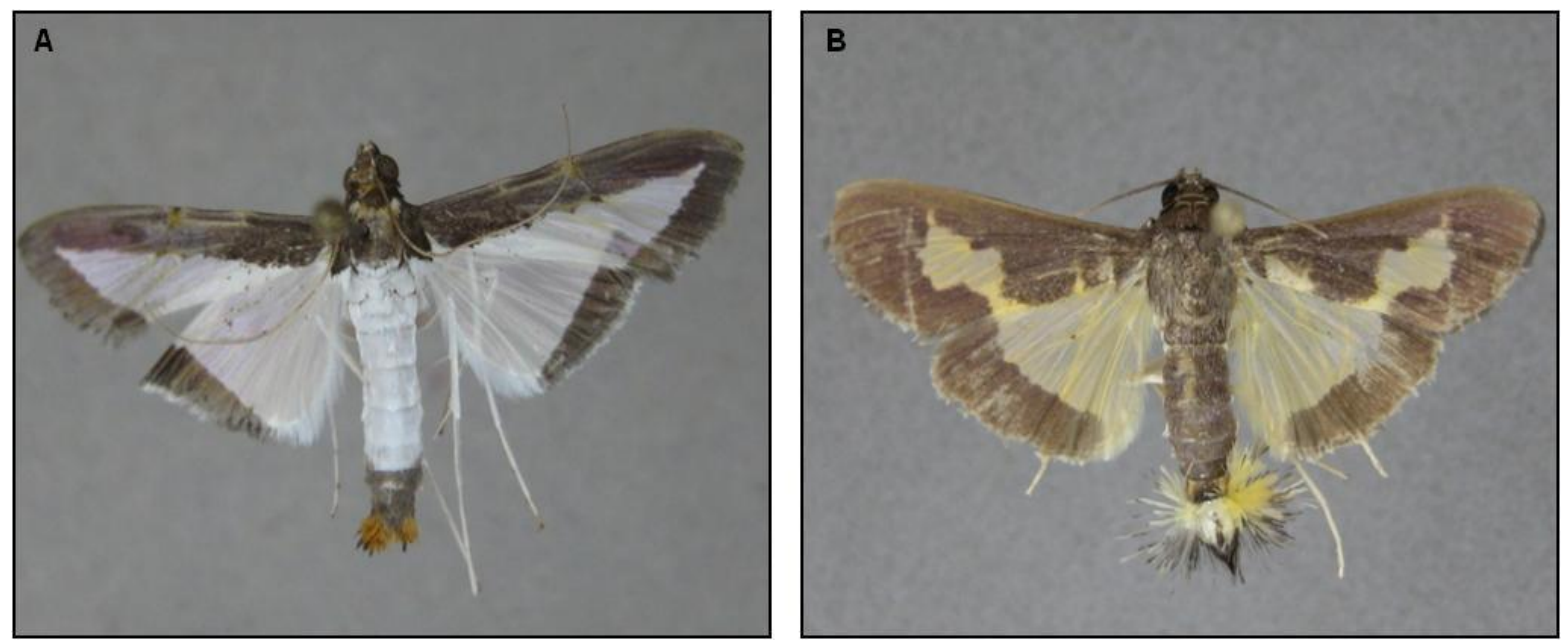

Figura 1. Adultos de Diaphania hyalinata (A) y D. ntidalis (B). (Fotos: E. Arcaya)

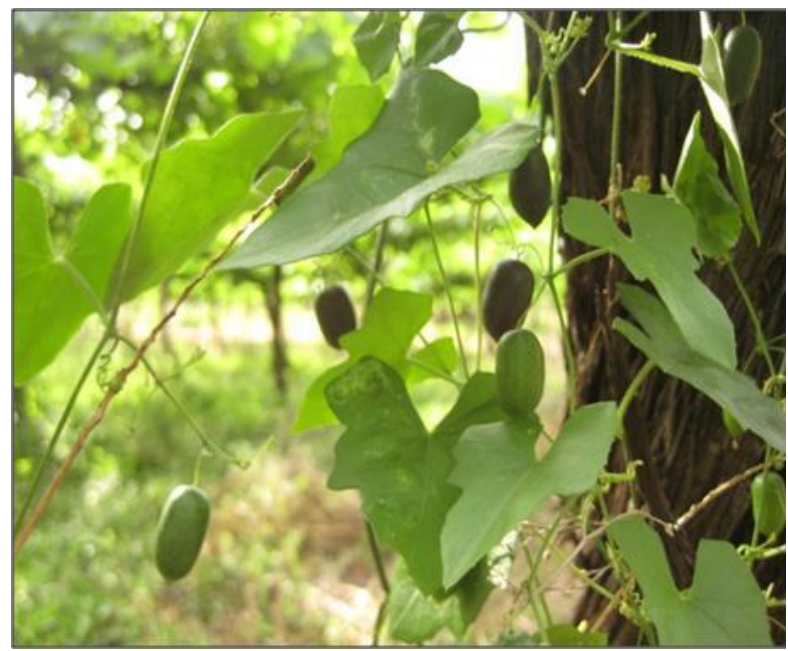

Figura 2. Pepino de culebra Melothria pendula L. (Cucurbitaceae). (Fotos: T. Capote)

La semejanza morfológica entre las tres especies es bien reconocida y no siempre son claramente identificadas por lo que son citadas en la literatura como Diaphania spp. ocasionando un subregistro de las especies (Delgado et al., 1997; Hidalgo y González, 2007). De acuerdo con Clavijo (1990) las especies $D$. nitidalis, $D$. hyalina y $D$. indica son muy semejantes por lo que las clasifican en el grupo "hyalinata". De la misma manera, el servicio de reconocimiento de plagas en puertos del Departamento de Agricultura de los Estados Unidos de América (USDA) agrupa a $D$. hyalinata y $D$. indica como hyalinata-indica complex debido a la dificultad para diferenciar a sus larvas (Gilligan \& Passoa, 2014). Un estudio de variabilidad isoenzimática con ejemplares de $D$. nitidalis, $D$. hyalinata y $D$. indica de tres localidades del estado Aragua, Venezuela, confirmó la relación de semejanza genética que existe entre $D$. hyalinata, $D$. nitidalis y $D$. indica, concluyéndose que el mayor grado de semejanza existe entre $D$. hyalinata y $D$. indica, siendo menor con D. nitidalis (Delgado, 2004). 
Es importante considerar que además de $M$. pendula como hospedante natural de Diaphania se han reportado como planta hospedante a Amaranthus dubius Mart.ex.Thell y A. viridis L. (Lenin, 2011). Las especies $A$. dubius y $A$. viridis son también referidas como malezas en cultivos de guayaba (Pacheco et al., 2009) y M. pendula como maleza en bananos (Rodríguez y Agüero, 2000) aspecto que debe ser considerado en la evaluación de otros factores que favorezcan la aparición de insectos plagas. Una característica fisiológica de $M$. pendula de importancia a considerar para el control como maleza y hospedante de Diaphania es que posee estructuras subterráneas perennes que le permiten retoñar en función de la disponibilidad de agua (Lira y Casa, 1998).

Se conoce que el control biológico de plagas por manejo "push-pull" que se basa en el empleo combinados de plantas atractivas (push) y repelentes (pull) de insectos en los cultivos (Paredes, Campos y Cayuela, 2013), dado que esta tecnología ha sido estudiada en melón para evaluar las preferencias de estados inmaduros de $D$. nitidalis para su control utilizando calabaza como cultivo trampa y sandía como barrera repelente (Leiner y Spafforf , 2016), sería de gran interés agronómico evaluar la capacidad de $M$. pendula como cultivo trampa.

Con este registro de $D$. hyalinata y $D$. nitidalis se confirma la presencia de las tres especies de importancia agronómica del grupo "hyalinata" (Clavijo, 1990) en el municipio Palavecino del estado Lara. En el aspecto económico, por ser un estado productor de rubros como el pepino y melón, el reporte de esta relación entre las especies de Diaphania y $M$. pendula constituye un factor de importancia a considerar cuando se desarrollen programas de control y ejecuten adecuadas prácticas culturales en los cultivos de cucurbitáceas en el estado Lara.

\section{AGRADECIMIENTOS}

Al Consejo de Desarrollo Científico, Humanístico y Tecnológico (CDCHT-UCLA) por el financiamiento del proyecto 1014-AG-2016. A la Profesora Sonia Piña del Instituto de la Uva, Decanato de Agronomía de la UCLA por permitir la recolección del material entomológico y al Dr. Quintín Arias del Museo del Instituto de Zoología Agrícola (MIZA) de la Facultad de Agronomía de la Universidad Central de
Venezuela (UCV), Maracay, estado Aragua por la confirmación de las especies de Diaphania.

\section{REFERENCIAS BIBLIOGRÁFICAS}

Acevedo, I., Marcano, C., Contreras, J., Jiménez, O., Escalona, A. y Pérez, P. (2012). Caracterización del manejo agronómico del cultivo de pepino (Cucumis sativus L.) en Humocaro Bajo, estado Lara, Venezuela. Revista Unellez de Ciencia y Tecnología, $30,36-42$.

Arcaya, E., Díaz, F. \& Paz, R. (2004). Primer registro de Diaphania indica (Saunders, 1851) (Lepidoptera: Cambridae) en el cultivo de pepino en Venezuela. Bioagro, 16(1), 73-74.

Arévalo, N.L. (2016). Evaluación de tres concentraciones de benzoato de emamectina en el control de Diaphania nitidalis (Lepidoptera, Pyralidae) en el cultivo de pepinillo (Cucumis sativus L.). Tesis Lic. Trujillo, Perú: Universidad privada Antenor Orrego, 68p.

Arias, Q. y Clavijo, J. (2001). Clave pictórica de las especies de Diaphania Hübner, 1818 (Lepidoptera: Crambidae) de Venezuela. Entomotropica, 16(1), 113.

Arzate-Fernández, A.M. y Grenón-Cascales, G.N. (2002). Contribución al conocimiento del pepinillo silvestre (Melothria pendula L.). Ciencia Ergo Sum 9(1), 78-86.

Brito, G.G. de, Costa, E.C., Maziero, H., Brito, A.B. é Dörr, F.A. (2004). Preferência da broca-dascucurbitáceas [Diaphania nitidalis Cramer, 1782 (Lepidoptera: Pyralidae)] por cultivares de pepineiro em ambiente protegido. Ciencia Rural, $34(2), 577-579$.

Brou, V.A. (2016). Diaphania hyalinata (Linnaeus) (Lepidoptera: Crambidae). Southern Lepidopterist's News, 38(4), 227-228. Consultado 22 feb. 2018. Disponible en: https://www.researchgate.net/publication/311407 566_Diaphania_hyalinata_Linnaeus_Lepidoptera_P yralidae_in_Louisiana.

Capinera, J.L. (2017a). Pickleworm Diaphania nitidalis (Stoll) (Insecta: Lepidoptera: Crambidae). Gainesville, Florida: University of Florida (Serie of the Department of Entomology and Nematology, UF/IFAS Extension, EENY-164). Consultado 22 feb. 
$2018 . \quad$ Disponible en: http://entnemdept.ufl.edu/creatures/veg/picklewor m.htm

Capinera, J.L. (2017b). Melonworm, Diaphania hyalinata Linnaeus (Insecta: Lepidoptera: Crambidae). Gainesville, Florida: University of Florida (Serie of the Department of Entomology and Nematology, UF/IFAS Extension, EENY-163). Consultado 04 abr. 2018. Disponible en: http://entnemdept.ufl.edu/creatures/veg/leaf/melo nworm.htm

Clavijo, J. (1990). Systematics of Black and White Species of the Genus Diaphania Hübner, 1818 (Lepidoptera: Pyralidae: Pyraustinae). Tesis PhD. Montreal, Canadá: Mc Gill University: Faculty of Agricu1tural and Environmental Sciences, pp. 274.

Clavijo, J., Munroe, E. y Arias, Q. (1995). El género Diaphania Hübner (Lepidoptera: Crambidae): clave para las especies de importancia económica. Agronomía Tropical, 45(3), 347-358.

Clavijo-Albertos, A. y Arias-Celis, Q. (2017). Catálogo de los Spilomelinae de Venezuela (Lepidoptera: Crambidae). SHILAP Revista de Lepidopterología, 45 (177), 129-141.

Cordo, H., Logarzo, G., Braun, K. y Di Lorio, O. (2004). Catálogo de Insectos Fitófagos de la Argentina y sus Plantas Asociadas, adaptación digital. Fundación para el Estudio de las Especies Invasivas. Buenos Aires, Argentina: Sociedad Entomológica Argentina ediciones. Consultado 19 febrero $2018 . \quad$ Disponible en: http://fuedei.org/catalogo/

Delascio-Chitty, F. y López R. (2007). Las cucurbitáceas del estado Cojedes, Venezuela. Acta Botánica Venezolana, 30(1), 19-41.

Delgado, A., Rojas, T., Carvajal, F., García, M., Arnal, E. y Rondón, A. (1997). Reconocimiento de insectos plagas y enfermedades en el estado Cojedes. Memoria-Sociedad de Ciencias Naturales La Salle, 17(147), 101-118.

Delgado, O. (2004). Variabilidad Isoenzimática de tres Especies de Diaphania (Lepidoptera) en Venezuela. Tesis Ing. Agr. Maracay, Venezuela: Universidad Central de Venezuela, Facultad de Agronomía, pp 71.
Elsey, K.D., Pena, J.E. \& Waddill, V.H. (1985). Suitalbility of Potencial Wild Hosts of Diaphania species in Southern Florida. Florida Entomologist, $68(4), 682-686$.

Flora Argentina. (2018). Plantas vasculares de la República Argentina: versión digital. Córdoba, Argentina: Instituto Multidisciplinario de Biología Vegetal. Consultado 18 febrero 2018. Disponible en: http://www.floraargentina.edu.ar/

Flora do Brasil. (2017). Flora do Brasil 2020 em construção. Rio de Janeiro, Brasil. Consultado 18 febrero 2018. Disponible en:

http://floradobrasil.jbrj.gov.br/reflora/listaBrasil/Pr incipalUC/PrincipalUC. do;jsessionid=E6AFAEE9FC6 E70D7E9CBB3D8D31B5727\#CondicaoTaxonCP

Geraud-Pouey, F., Chirinos, D., Peña, M. y Arcila, S. (1998). Artrópodos asociados con el cultivo del melón en la zona noroccidental de Venezuela. Revista de la Facultad de Agronomía, 15, 11-22.

Gilligan, T.M. \& Passoa, S. (2014).

LepInterceptept: a identification resource for intercepted Lepidoptera larvae. Fort Collins, USA: Colorado State University. Consultado 19 feb. 2018. Disponible en:

http://idtools.org/id/leps/lepintercept/indica.html

Hardy, I. (1976). Consideraciones Generales sobre Frutos y Semillas de las Cucurbitaceae en Venezuela. Acta Botánica Venezolana 11(1-4), 205281.

CICY (Centro de Investigación Científica de Yucatán). (2018). Flora de la Península de Yucatán. Mérida, Yucatán, México. Consultado 18 feb. 2018. Disponible en: http://www.cicy.mx

Hidalgo, P. y González, R. (2007). Manual práctico para la producción de semilla artesanal de pepino. Maracay, Venezuela: Instituto Nacional de Investigaciones Agrícolas, $92 \mathrm{p}$.

Hokche, O., Berry, P.E. y Huber, O. (2008). Nuevo Catálogo de la Flora Vascular de Venezuela. Caracas, Venezuela: Fundación Instituto Botánico de Venezuela, $859 \mathrm{p}$.

ICN (Instituto de Ciencias Naturales). (2017). Colecciones en línea. Bogotá, Colombia: Instituto de Ciencias Naturales: Universidad Nacional de Colombia. Consultado 18 feb. 2018. Disponible en http://www.biovirtual.unal.edu.co/ 
Janzen, D.H. \& Hallwachs, W. (2009). Dynamic database for an inventory of the macrocaterpillar fauna, and its food plants and parasitoids, of Area de Conservacion Guanacaste (ACG), northwestern Costa Rica. Guanacaste, Costa Rica. Consultado 01 mar. 2018.2 Disponible en: http://janzen.sas.upenn.edu

Leiner, R. \& Spafford, H. (2016). Pickleworm (Diaphania nitidalis Cramer) Neonate Feeding Preferences and the Implications for a Push-Pull Management System. Insects, 7(32), 1-11.

Lenin, N. (2011). Infestation of the pumpkin caterpillar, Diaphania indica Saunders in cucurbits and its management. Tesis MSc. Kerala, India: Agriculture Kerala Agricultural University, 120 p.

Lira, R. (2001). Cucurbitaceae. Michoacán, México, Instituto de Ecología A.C., Serie Flora del Bajío y regiones adyacentes, 120p. Consultado 24 feb. $2018 . \quad$ Disponible en http://www1.inecol.edu.mx/publicaciones/resumen ess/FLOBA/Flora\%2092.pdf .

Lira, R. y Casa, A. (1998). Uso y manejo de Ibervillea millspauchll (Cogn.) C. Jeffrey, Melothria pendula L. y otras especies silvestres de la familia cucurbitaceae: posibles procesos de domesticación incipiente. Boletín de la Sociedad Botánica de México, 62, 77-89.

Olmo, H. (1968). The Potential for a Grape and Wine Industry in Venezuela. Caracas, Venezuela: Consejo de Bienestar Rural, $30 \mathrm{p}$.

Pacheco, D., Zambrano, O., Sthormes, G., Sánchez, A., Pettit, Y., Quirós, G.M. de...Dorado, I. (2009). Reconocimiento de malezas presentes en el huerto de guayabo (Psidium guajava L.) tipo Criolla Roja, del Centro Frutícola del Zulia, Municipio Mara, Venezuela. Rev. UDO Agri., 9(1), 141-147.

Paredes, D., Campos, M. y Cayuela, L. (2013). El control biológico de plagas de artrópodos por conservación: técnicas y estado del arte. Ecosistemas, 22(1), 56-61.
PYBIO (Paraguay Biodiversidad). (2018). Crambidae. Asunción, Paraguay, ParaguayBiodiversidad. Consultado 18 mar. 2018. Disponible en: http://www.pybio.org/index.php

Rodríguez, A.M. y Agüero, R. (2000). Identificación de malezas trepadoras del banano (Musa sp.) en la zona caribe de Costa Rica. Agronomia Mesoamericana, 11(1), 123-125.

Ruíz, C.A. y Russián, T. (2017). Melón: cultivo y poscosecha en la península de Paraguaná. Maracay, Venezuela: Instituto Nacional de Investigaciones Agrícolas, $112 \mathrm{p}$.

Salas, J.A. (2011). Compartiendo nuestras experiencias en investigación participativa, Caso: Cultivo del melón en San José de los Ranchos. Maracay, Venezuela: Instituto Nacional de Investigaciones Agrícolas, 31p.

Saunders, J.L., Coto, D.T. y King, A.B.S. (1998). Plagas Invertebradas de Cultivos Anuales Alimenticios de América Central. 2da edición, Turrialba, Costa Rica: CATIE. 305p. (Serie Manual Técnico, 29).

Schnee, L. (1984). Plantas comunes de Venezuela. $3^{\text {ra }}$ edición. Caracas, Venezuela: Universidad Central de Venezuela, Ediciones de la Biblioteca, 806p. (Serie Colección Ciencias Biológicas).

The Plant List. (2013). The Plant List: a working list of all plant species. Reino Unido: Real Jardín Botánico de Kew. Consultado 18 mar. 2018. Disponible en: http://www.theplantlist.org/

USDA (Natural Resources Conservation Service). (2018). Plants database. Estados Unidos de América: United States Department of Agriculture. Consultado 18 feb. 2018. Disponible en: https://plants.usda.gov/core/profile?symbol=mepe 3.

VanDick, J. (2018). GugGuide.net. Iowa, Estados Unidos de América: Iowa State University, Department of Entomology. Consultado 18 feb. 2018. Disponible en: https://bugguide.net 